\title{
Research on Design Theory of Cam Type Torsional Transmission Mechanism
}

\author{
Fanbi Wang, Tieshan Zhang ${ }^{*}$ and Mingsong Li \\ College of mechanical eng., Nanjing Univ. of Science and Technology, Nanjing 210094, China \\ *Corresponding author
}

\begin{abstract}
Putting forward a cam mechanism, which realize torsion moment transfer. Firstly, establishing the mechanical model of the mechanism. According to the principle of geometry, the corresponding mathematical model is established, which based on the analysis of the mechanical model. Through analysis of mathematical model, the transfer law of this torsion moment is determined by the normal load shape function and tangential load shape function, which is about the torsion moment of this torsion drive mechanism, numerical size of shape function depends on the cam rotor diameter and the stiffness of elastic component. The simulation results of the mechanism simulated by the simulation tool agree with the results of the theoretical Equations established by the author, which prove that the design Equations are credible.
\end{abstract}

Keyword-torsion drive, cam mechanism, design theory

\section{INTRODUCTION}

Mechanical transmission is one of the chief components of the vehicle and other mechanical systems, in which there are gear drive, chain drive, friction drive, etc. Whatever form of transmission, there are the problems of vibration and noise, for rotation, there exists torsional vibration and noise problem.

In order to reduce torsional vibration and noise, scholars in the field have done a lot of research. In vehicle engineering, in order to reduce the vibration and noise of the transmission system, researchers developed clutch torsional vibration damper, double mass flywheel and other mechanisms, which have improved the problems in engineering[1].

However, there are some problems for the various devices in use[2], therefore, it is need for new solutions of the torsional vibration issue. The author will discuss the new solution in the research of this paper.

\section{MECHANICAL MODEL}

In order to solve torsional vibration problems, most of vehicle mechanical transmission system are installed clutch torsional vibration damper. As its performance is not good enough, the double mass flywheel is being used to replace the torsional vibration damper in some vehicle mechanical transmission system, and the better result is obtained. However, there are a series of problems in the transmission system of the dual mass flywheel, which include structural complexity, Long arc spring deformation by centrifugal force affecting torsion damping performance and other issues. The author puts forward using the mechanism transfer torque as shown in Figure I.In the
Fig.1, Outer rotor and inner rotor are linked by an elastic component. When the outer rotor rotation through the elastic component to rotate the inner rotor, torque transmission can be realized. The spin axes of the inner and outer rotor are both through point $\mathrm{O}$ and perpendicular to the plane.

The end of elastic component 3 is fixedly connected with the outer rotor, and the other end contacted with the inner rotor. In the initial position, the preload acting on the inner rotor passes through the rotation axis of the rotor and therefore does not generate torque. When the elastic component rotates with the outer rotor, The force of elastic element acting on the inner rotor is not through the rotation axis, therefore, it will generate torque and drive the inner rotor run.

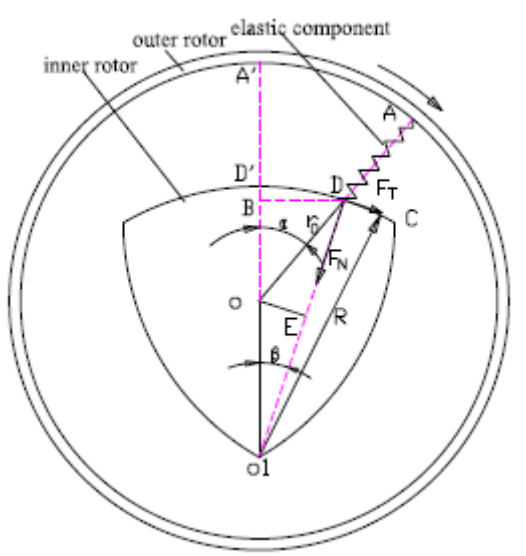

FIGURE I. MECHANICAL MODEL OF CAM MECHANISM

The elastic component force acting on inner rotor is composed of two parts, one part is the normal force of the elastic component, the other part is the friction force corresponding to the normal force of the elastic component. When the outer rotor rotates one angle with respect to the inner rotor, the normal force of the elastic component can be resolved into the component along the surface normal direction and the surface tangential direction of the inner rotor.

By analyzing the mechanical model, the greater the relative angle of inner and outer rotor, the greater the elastic deformation of the elastic component. In other words, the force along a normal direction of the inner rotor is increasing owing to the elastic force increases, at the same time, the greater the arm of force. According to the mechanical model of Figure I, the maximum relative rotation angle of the two rotors is 60 degrees. 
The tangential force becomes larger with the increase of the relative rotation angle of the inner and outer rotor, but little change in the arm of force. The friction force corresponding to the elastic component is along the tangential direction of the inner rotor, and its size is related to the normal force and the friction coefficient.

\section{MATHEMATICAL MODEL ESTABLISHMENT}

It is necessary to establish the mathematical model on the basis of the analysis of mechanical model to determine whether the mechanical model of the mechanism can meet the requirements of mechanical transmission. At the same time, it is needed to establish the corresponding mathematical model to study the ability of the mechanism to transfer the torque. Therefore, writers will discuss the mathematical model of the mechanism shown in Fig.1.

\section{A. Cam Surface Normal Load}

The triangle cam is divided into three equal parts along the circumferential direction. Therefore, only the $1 / 3$ cam is studied, the calculated torque multiplied by three is the total torque. Force on the cam surface which normal direction upward will be analyzed in the following analysis. Assume the rotary center of the cam as the $\mathrm{O}$ point, the angular point of the cam as the $\mathrm{O}_{1}$ point, the contact point of the elastic element and the cam as D point, the projection point of point $\mathrm{D}$ on the line $\mathrm{OO}_{1}$ as $\mathrm{B}$ point. Point $\mathrm{O}_{1}, \mathrm{D}, \mathrm{B}$ and $\mathrm{O}, \mathrm{D}, \mathrm{B}$ respectively constitute two triangles $\Delta \mathrm{O}_{1} \mathrm{DB}$ and $\triangle \mathrm{ODB}$. In $\Delta \mathrm{O}_{1} \mathrm{DB}, \mathrm{BD}=\mathrm{R} \sin \beta$, $O_{1} B=R \cos \beta$.According to the nature of the triangle, $\mathrm{O}_{1} \mathrm{O}=\sqrt{3} / 3 \mathrm{R}$ can be deduced, and in $\Delta \mathrm{ODB}, \mathrm{BD}=\mathrm{R} \sin \beta, \mathrm{OB}=\mathrm{R} \cos \beta-\sqrt{3} / 3 \mathrm{R}$.Thus the following equation can be derived out

$$
\mathrm{OD}=\sqrt{\mathrm{BD}^{2}+\mathrm{OB}^{2}}=\mathrm{R} \sqrt{4 / 3-2 \sqrt{3} / 3 \cos \beta} .
$$

According to the triangle theorem: $\quad \sin \alpha=\mathrm{BD} / \mathrm{OD} \quad, \quad \sin \beta=\mathrm{BD} / \mathrm{R}$, therefore $\sin \alpha / \sin \beta=\mathrm{R} / \mathrm{OD}$

Put it into the Eq.1

$$
\sin ^{2} \alpha(4 / 3-2 \sqrt{3} / 3 \cos \beta)=\sin ^{2} \beta .
$$

The relationship between $\alpha$ and $\beta$ can be derived from the Eq.2, as shown in the following:

$$
\beta=\arccos \beta=\arccos \left(\sqrt{3} / 3 \sin ^{2} \alpha+\sqrt{1-4 / 3 \sin ^{2} \alpha+1 / 3 \sin ^{4} \alpha}\right)
$$

When the elastic component is driven by the outer rotor from position $\mathrm{A}^{\prime} \mathrm{D}$ ' to $\mathrm{AD}$, the elastic component is compressed and the compression length is $\Delta \mathrm{H}$.

$$
\Delta \mathrm{H}=\mathrm{A}^{\prime} \mathrm{D}^{\prime}-\mathrm{AD}=\mathrm{R} \sqrt{4 / 3-2 \sqrt{3} / 3 \cos \beta}-\mathrm{R} \sqrt{4 / 3-2 \sqrt{3} / 3}
$$

The elastic component normal load along the cam surface can be derive:

$$
\mathrm{F}_{\mathrm{N}}=\Delta \mathrm{HK} \cos (\alpha-\beta)=\mathrm{F}_{\mathrm{t}} \cos (\alpha-\beta) .
$$

In the above Equation, $F_{t}$ is elastic force of the elastic component.

The arm OE of normal load corresponding the center $\mathrm{O}$ is: $\mathrm{OE}=\sqrt{3} / 3 \mathrm{R} \sin \beta$.

And then, the torque $\left(\mathrm{M}_{1}\right)$ of the elastic component normal load acting on the cam will be obtain:

$\mathrm{M}_{1}=\mathrm{F}_{\mathrm{N}} \times \mathrm{OE}=\sqrt{3} / 3 \mathrm{R}^{2} \mathrm{~K} \sin \beta \cos (\alpha-\beta) \times(\sqrt{4 / 3-2 \sqrt{3} / 3 \cos \beta}-\sqrt{4 / 3-2 \sqrt{3} / 3})$

Because the cam mechanism has three elastic component, so the total moment of elastic component normal load of cam is:

$$
M_{n T}=3 \times M_{1}=\sqrt{3} R^{2} K \sin \beta \cos (\alpha-\beta) \times(\sqrt{4 / 3-2 \sqrt{3} / 3 \cos \beta}-\sqrt{4 / 3-2 \sqrt{3} / 3})
$$

\section{B. Transmission Mechanism Normal Load Shape Function}

As can be seen from the Eq.5, the torque transfer capability of the mechanism is proportional to the torsional stiffness $\mathrm{K}$ of the elastic component, and is proportional to $\mathrm{R}^{2}$ (define $\mathrm{R}$ as the curvature radius of the cam surface).In addition, $\mathrm{S}$ of the Eq.6 also affects the torque. Once the angle $\alpha$ of outer rotor relative to inner rotor was determined, the value of the $\mathrm{S}$ can be determined. $S$ curve shape determines the shape of the angle of twist and torque curve, that is $\mathrm{S}$ determines the law of the torsional moment of the normal load. $\mathrm{S}$ is named normal load shape function.

$$
S=\sin \beta \cos (\alpha-\beta) \times(\sqrt{4 / 3-2 \sqrt{3} / 3 \cos \beta}-\sqrt{4 / 3-2 \sqrt{3} / 3}) .
$$

Eq. 3 and Eq. 6 show that the normal load shape function is the function of $\alpha$,and the graph of normal load shape function from $\alpha=0$ to $\alpha=60^{\circ}$ is shown in Figure II . Obviously, the shape of the curve indicates the mechanism is in line with the requirements of the general torsion drive system.

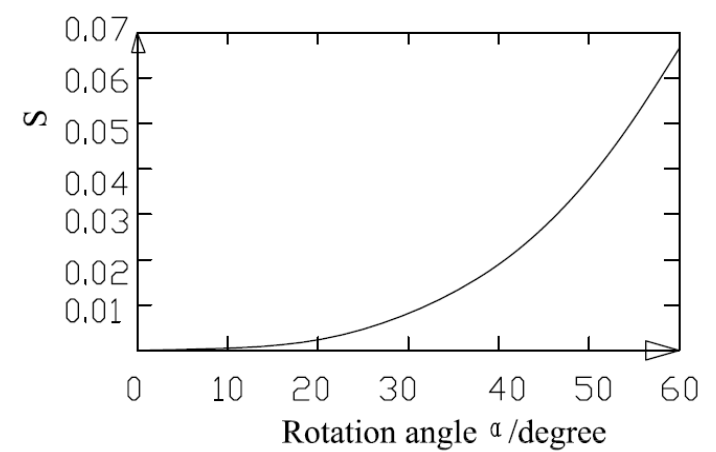

FIGURE II. THE CURVE OF NORMAL LOAD SHAPE FUNCTION 


\section{Cam Surface Tangential Load and Transmission \\ Tangential Load Shape Function}

The tangential force along the cam surface generated by the deformation of a single elastic component is:

$$
F_{q}=F_{t} \cdot \sin (\alpha-\beta)
$$

The arm of the tangential force corresponding the center $\mathrm{O}$ is $\mathrm{L}$

$$
\mathrm{L}=\mathrm{OD} \times \cos (\alpha-\beta)
$$

The corresponding moment $\mathrm{M}_{\mathrm{q}}$ is:

$$
\mathrm{Mq}=\mathrm{F}_{\mathrm{q}} \times \mathrm{L}=\mathrm{F}_{\mathrm{t}} \cdot \sin (\alpha-\beta) \times \mathrm{OD} \times \cos (\alpha-\beta) \text {. }
$$

Three elastic components produce total moment of tangential force is:

$$
\mathrm{M}_{\mathrm{qT}}=3 \times \mathrm{F}_{\mathrm{q}} \times \mathrm{L}=3 \times \mathrm{F}_{\mathrm{t}} \cdot \sin (\alpha-\beta) \times \mathrm{OD} \times \cos (\alpha-\beta)=3 \mathrm{~F}_{\mathrm{t}} \times \mathrm{OD} \times \mathrm{q}
$$

In the formula:

$$
q=\sin (\alpha-\beta) \cdot \cos (\alpha-\beta)
$$

There is still friction between the elastic element and the cam type surface contact, so the friction force is needed to deduce. The elastic force $F_{m}$ of the elastic component on the cam surface friction is:

$$
\mathrm{F}_{\mathrm{m}}=\mathrm{F}_{\mathrm{t}} \cdot \cos (\alpha-\beta) \times \mathrm{f}
$$

The friction torque $\mathrm{M}_{\mathrm{m}}$ generated by the deformation of a single elastic component is:

$$
\mathrm{M}_{\mathrm{m}}=\mathrm{F}_{\mathrm{t}} \cdot \mathrm{OD} \times \mathrm{f} \times \cos (\alpha-\beta)^{2} .
$$

The total friction moment is

$$
\mathrm{M}_{\mathrm{mT}}=3 \times \mathrm{F}_{\mathrm{t}} \times \mathrm{OD} \times \mathrm{f} \times \cos (\alpha-\beta)^{2}=3 \mathrm{~F}_{\mathrm{t}} \times \mathrm{OD} \times \mathrm{m} .
$$

In the formula:

$$
\mathrm{m}=\mathrm{f} \times \cos (\alpha-\beta)
$$

When designing the mechanism, generally keep the elastic component and the cam surface in good contact condition, that is, the friction coefficient maintains very small state. In order to view the changes of $\mathrm{q}$ and $\mathrm{m}$, setting the friction coefficient $\mathrm{f}=$ 0.05.According to Eq.3, Eq.10, Eq.13, the results of the calculation as shown in Figure III.

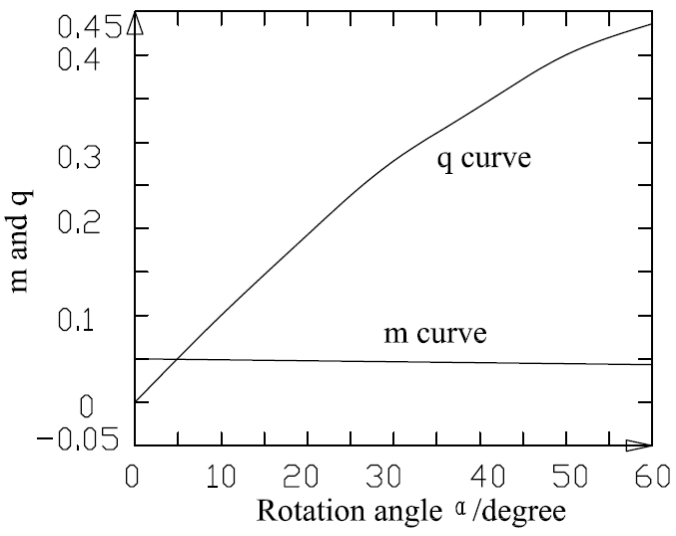

FIGURE III. FIGURE 3. TANGENTIAL LOAD FUNCTION

The change law of $m$ and $q$ along with angle $\alpha$ is shown in Fig. 3.m and q is described by Eq.10, Eq.13, and they are called tangential load shape functions.

The total torque generated by the tangential load (including the friction force) produced by elastic component acting on cam surface is:

$$
\mathrm{M}_{\mathrm{mqT}}=\mathrm{M}_{\mathrm{mT}}+\mathrm{M}_{\mathrm{qT}} \text {. }
$$

\section{The Total Torque}

According to the results of Section 2.1 to 2.3, the total torque of the mechanism is composed of the moment generated by the normal load and the tangential load. Among them, the torque generated by the normal load is the main part of the total torque. The total torque is:

$$
\mathrm{M}_{\mathrm{T}}=\mathrm{M}_{\mathrm{nT}}+\mathrm{M}_{\mathrm{mqT}} .
$$

\section{Simulation OF Mechanical Model}

The author sets some basic parameters [1] of the mechanism as an example, adopting Eq.1 to Eq.15 to calculate, and the result is shown in Fig.4. As it can be seen from Fig. 4, the relationship between the angle of twist and torque transmission accords with the demand of the vehicle and some other systems. At the same time, the author uses multi-body system dynamics software to simulate the mechanism, the result as shown in Figure IV.

From the results of the two methods, it can be seen that the law of the two are consistent , the numerical difference is small, which also shows that the relevant design equations deduced by the author are correct. 


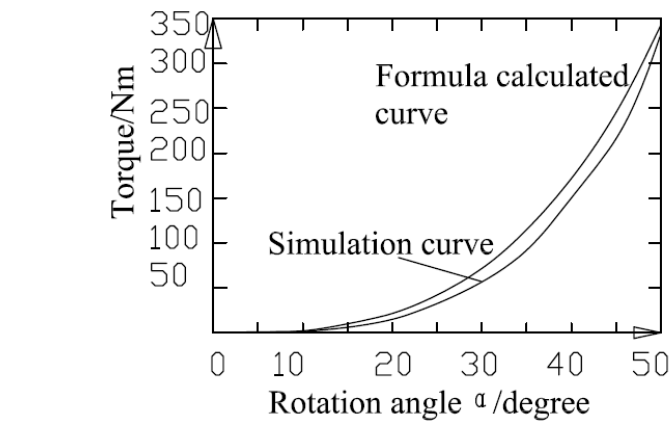

FIGURE IV. COMPARISON OF SIMULATION RESULTS AND THEORETICAL VALUES

\section{SUMMARY}

1) The cam type torsional transmission mechanism which is composed of the outer rotor, the elastic component and the triangular cam (inner rotor) can transfer torsion moment according to a certain law.

2) According to the geometrical relationship of the mechanism, the torque transfer relationship can be solved by the theory.

3) The torque transfer law of the cam type torsional transmission mechanism is determined by the normal load shape function and the tangential load shape function. The torque value depends on the rotor diameter.

\section{REFERENCES}

[1] Guang Ming Zhao, Study on nonlinear torsional vibration damping characteristics of the circumferential arc spring dual mass flywheel, Wuhan University Of Technology, Wuhan , 2013

[2] TieShan Zhang. MingSong Li ,A Design Study on the Torsion Vibration of the Vehicle Powertraint,Applied 5th International Conference on Advanced Engineering Materials and Technology(AEMT’2015),3(2015) 429-433. 